\section{Conclusions}

It is well recognized that the distribution of antibiotics varies between species; nevertheless, we believe that our observations have several therapeutic implications.

(1) The blood peak gives little indication of the tissue peak, which was often less than $50 \%$ of the blood peak. It is possible that despite a high blood peak the tissues may never be exposed to an adequate concentration of the drug. Conversely, an antibacterial agent with a low but prolonged peak may have adequate tissue fluid concentrations. Since these concentrations are unpredictable there appears to be no substitute for direct measurement in the study of the distribution of drugs in body fluids.

(2) In order to achieve effective tissue levels in clinical practice most antibacterial agents which are excreted rapidly should be administered either frequently or in sufficiently large doses to ensure that adequate blood levels are maintained and the tissue levels equilibrate. Maintenance of adequate blood levels may be particularly important for the distribution of these drugs in diseased tissues.

(3) Many drugs have negligible blood levels although they are widely used for the treatment of urinary tract infections. Most of these drugs are excreted mainly by glomerular filtration, and the therapeutic effectiveness of such drugs is dependent on the very high drug concentrations in the urine. However, it must be emphasized that the tissue concentrations in these circumstances are, like those in the blood, also negligible. Thus in the treatment of so-called tissue infections, and especially the tissues of the urinary tract, the choice of drug should be guided not only by the excellence of the urinary concentration but also by the ability to achieve adequate blood levels.

We wish to thank Mr. D. Martin, Miss Lynn Boardman, and Mr. L. M. Taylor for their expert technical help, and the department of medical illustration for the figures. We are grateful for the support for these investigations from Antibioticos S. A., Beecham Research Laboratories, British Schering Ltd., Eli Lilly and Co. Ltd., Roche Products Ltd., and the Wellcome Foundation.

\section{References}

Brown, D. M. (1964). Postgraduate Medical fournal, 40, December Suppl.,

p. 31. S. Ford, P. M., Holt, P. J. L., and Pflug, J. J. (1972a). British
Calnan, J. S., Fo

Fournal of Plastic Surgery, 25, 164. ., D., and Taylor, L. M. (1972b). Proceedings of the Royal Society of Medicine, 65, 715.

Chisholm, G. D., Calnan, J. S., and Waterworth, Pamela M. (1968). In Urinary Tract Infection, ed. F. O'Grady and W. Brumfitt, p. 194. London, Oxford University Press.

Cockett, A. T. K., Moore, R. S., and Roberts, A. P. (1967). Investigative Urology, 5, 250.

Currie, G. A., Little, P. J., and McDonald, S. J. (1966). Nephron, 3, 282.

Guyton, A. C. (1963). Circulation Research, 12, 399.

Lithander, A., Werner, B. O. A., and Forsgren, L. O. (1968). Antimicrobial Agents and Chemotherapy, 8, 366.

Orsolini, P. (1970). Postgraduate Medical fournal, 46, October Suppl., p. 13. Orsolini, P. (1970). Postgraduate Medical fournal, 46, October

Raeburn, J. A. (1971). Fournal of Clinical Pathology, 24, 633. inson, G. N. (1967). In Recent Advances in Medical Microbiology, ed.

A. P. Waterson, p. 254. London, Churchill. M. (1965). Medicine, 44, 1.
Stamey, T. A., Govan, D. E., and Palmer, J. M.

\title{
New Index for Evaluation of Oral Glucose Tolerance Test Results
}

\author{
W. Z. BILLEWICZ， J. ANDERSON， T. LIND
}

British Medical fournal, 1973, 1, 573-577

\section{Summary}

A simple numerical index is presented which describes the shape of an oral glucose tolerance response curve and appears to be a measure of the efficiency of homeostatic control. It is unafiected by the laboratory method of glucose estimation, the site of blood sampling, the amount of glucose given, and whether or not the patient has fasted overnight. The index satisfactorily differentiates clinically diabetic from non-diabetic individuals and seems to provide a sensitive method of describing small changes in glucose homeostatic control such as might occur, for example, in pregnancy.

\section{Introduction}

The recommendations by authoritative bodies such as the British Diabetic Association (Fitzgerald and Keen, 1964) and W.H.O. (1965) specify arbitrary concentrations of glucose at

M.R.C. Reproduction and Growth Unit, Princess Mary Maternity Hospital, Newcastle upon Tyne NE2 3BD

W. Z. BILLEWICZ, M.SC., Statistician

T. LIND, M.B., M.R.C.O.G., Consultant Obstetrician

Department of Medicine, University of Newcastle upon Tyne J. ANDERSON, M.B., M.R.C.P., Senior Lecturer given times after an oral glucose load, above which the response should be regarded as "abnormal."

Apart from the fact that criteria based on absolute levels or on increments of blood glucose may require adjustment in accordance with the source of the blood and whether determinations are made on whole blood or plasma (Lind et al., 1972), the essentially all-or-none nature of the most widely used criteria does not allow accurate description of relatively small changes such as might occur, for example, in normal pregnancy or after medication with, say, oral contraceptive or hypoglycaemic agents.

The total area under the curve provides one simple single figure method of describing the changes in blood glucose after an oral load. That method was used by Jarrett and Graver (1968) to describe changes occurring during the course of the menstrual cycle. The total area, however, is heavily influenced by the portion under the fasting blood glucose level so that a small change in the level has a disproportionately large effect.

Most of the body's control systems, like those in engineering, operate by means of a closed loop or "feedback" mechanism so that displacement from a preset norm, whether for temperature, $\mathrm{pH}$, osmolality, or plasma glucose concentration, brings corrective measures into play which drive the value back towards the norm. When using plasma glucose as an example a rise in concentration or positive "error" invokes corrective measures including the secretion of insulin. And since no control system is perfect the concentration tends to be driven below the norm, a negative error, which in turn invokes mechanisms to send the glucose concentration up again. Even in the fasting state such "hunting" around the norm can be detected, 
and when a large positive displacement takes place after a meal a considerable time is needed for the ripples to die away. In these circumstances the excursion of the plasma glucose concentration after an oral load resembles the harmonic oscillation of a damped spring which has been displaced. The relation between the amplitude and the time taken for the oscillations to disappear is indicative of the efficiency of the control system.

An approach to the analysis of blood glucose curves after a glucose meal, based on those principles, has been used by Ceresa et al. (1962) and also by Ackerman et al. (1964), who suggested that the natural (undamped) period of oscillation $\left(\omega_{0}\right)$ might provide a useful means of assessing the response and found that periods in excess of four hours indicated impaired control. Unfortunately, the determination of $\omega_{0}$ from curves fitted to test values is so mathematically complex that it is unlikely to be of general value to clinicians.

In this paper we describe a simple arithmetical method of describing the "shape" of an oral glucose tolerance test (O.G.T.T.) response curve, yielding a single figure index, $\mathrm{H}$, which expresses the efficiency of homeostatic control and which correlates very well with values of $\omega_{0}$ obtained by Ackerman's method $(r=0.86)$. It has several practical advantages in addition to its mathematical simplicity. Preliminary investigations suggest that it may provide a useful tool for the clinical evaluation of O.G.T.T. results and for research.

\section{Determination of the $H$ Index}

In terms of ordinary clinical experience, and in line with what we have said about homeostatic control, "good" glucose tolerance may be typified in descriptive terms by a blood glucose curve which is allowed to rise for only a short time before being rapidly returned to the fasting level一that is, the "peak" glucose level occurs early and the raised glucose level occurs for only a short time. Conversely, "bad" glucose tolerance is typified by a blood glucose which is allowed to continue rising for much longer-a delayed peak-and which stays longer above the fasting level.

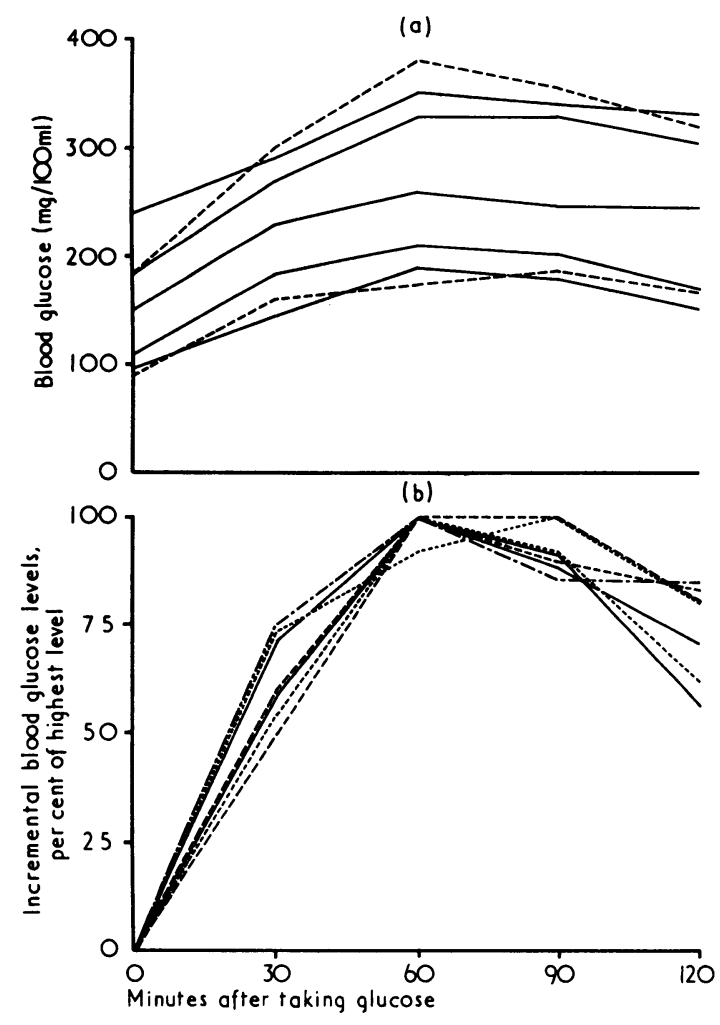

FIG. 1-Actual (a) and simplified (b) shapes of glucose curves corresponding to $\mathrm{H}$ values between 10 and 14.9
Analysis of the shape of the blood glucose response curve should take account of these two main features: the length of time blood glucose is allowed to go on rising (the time to peak), and the degree to which it returns towards fasting during the test period. The index we propose is based on a test period of two hours and takes account of both. The time to peak value is easily calculated and the extent of any prolonged rise of the blood glucose is described by the ratio of the incremental area for two hours to the incremental area for the first hour. The equation takes the form:

$$
\mathrm{H}=\text { Time to peak } \times \begin{aligned}
& \text { area above fasting level }(0-2 \mathrm{hr}) \\
& \text { area above fasting level }(0-1 \mathrm{hr})
\end{aligned}
$$

The method of calculating the necessary values is described in the Appendix.

It is obvious, from the formula, that later peaks and higher ratios of the two-hour to the one-hour incremental areas result in larger values of $H$, and vice versa. By using the method of calculation described in the Appendix values of $H$ ranging from two to 30 were obtained from a series of O.G.T.T. results covering a range from the obviously normal to the obviously abnormal.

To illustrate the manner in which values of $\mathrm{H}$ indicate the shape of an O.G.T.T. response we have chosen seven curves which yielded $H$ values from 10 to 14 (fig. 1a). That these seven curves have essentially similar shapes is shown in fig. $1 \mathrm{~b}$, in which the incremental values have been expressed as percentages of the highest observed increments. The average shapes shown in fig. 2 (scaled in the same way as fig. 1b) were obtained for various values of $H$, from under 3 to 20 or more.

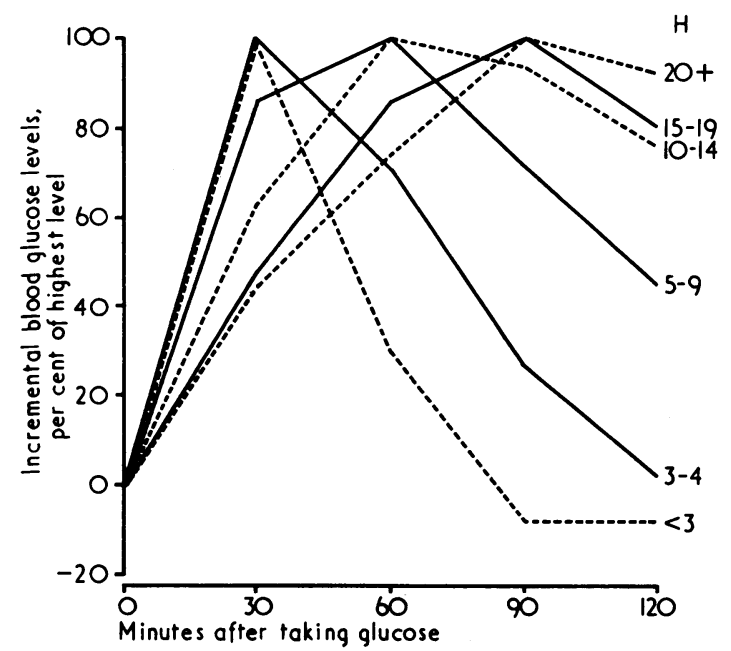

FIG. 2-Simplified shapes of glucose curves corresponding to various ranges of $\mathrm{H}$ values.

\section{The $\mathbf{H}$ Index in Practice}

\section{CLINICAL INTERPRETATION}

The index was calculated for O.G.T.T.s from 78 subjects36 normal, healthy, non-pregnant women aged $20-45$, and 22 men and 20 women in the age range 34-80 who were suspected of having diabetes mellitus but who had not been given insulin up to the time of the test. In all cases blood samples were taken after fasting overnight and $30,60,90$, and 120 minutes after taking $50 \mathrm{~g}$ glucose by mouth. The subjects were divided into three categories.

Diabetic.-According to the definition of the British Diabetic Association (Fitzgerald and Keen, 1964)-that is, a peak concentration of at least $180 \mathrm{mg} / 100 \mathrm{ml}$ (venous blood), plus a two-hour concentration of at least $120 \mathrm{mg} / 100 \mathrm{ml}$ (capillary blood) or $110 \mathrm{mg} / 100 \mathrm{ml}$ (venous blood). Cases not diabetic by that definition were arbitrarily divided into the following two categories. 
Suspect.-Subjects with one of the two abnormalities specified for diabetes.

Normal.-Subjects with neither of the abnormalities.

That the index discriminates well between normal and diabetic subjects is shown in fig. 3 . Of the 36 normal cases, $94 \%$ had values of $\mathrm{H}$ under 5; all the 33 diabetic cases had values of 5 or more, and $73 \%$ had values of 10 or more. The nine suspect cases had values ranging from 4 to 10 . On the basis of that evidence we suggest that the following classification of $\mathrm{H}$ index values may be found useful in clinical contexts: normal, under 5; suspect, 5-9; abnormal, 10 and over.

Whether these criteria remain valid in other conditions, such as pregnancy, in which many aspects of homeostasis are altered remains to be seen.

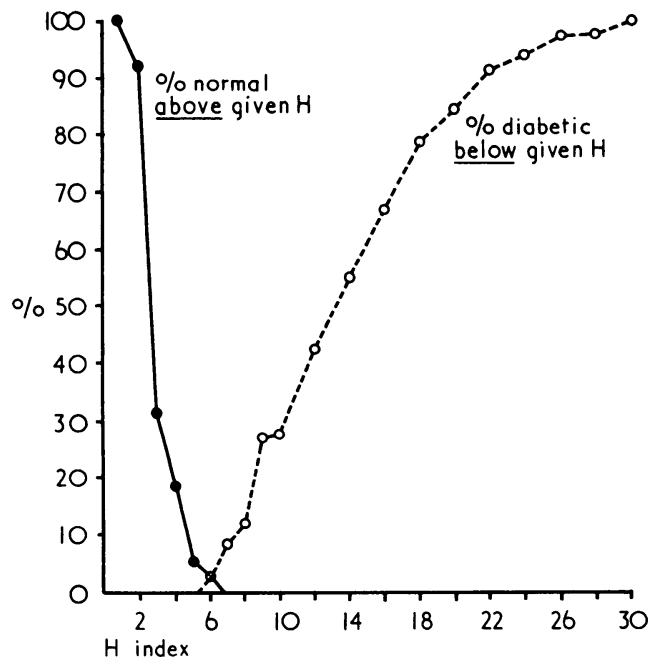

FIG. 3-Distribution of $H$ values for persons classified as normal and persons classified diabetic on the basis of an oral glucose tolerance test. Patients classified as suspect had values ranging from 4 to 10 .

\section{TECHNICAL ADVANTAGES}

Whole Blood or Plasma.-Glucose concentration was estimated in both whole blood and its plasma in 100 O.G.T.T.s on pregnant women, not otherwise reported in this paper. For pairs of $\mathrm{H}$ indices on this basis the correlation coefficient was 0.98 , indicating that this source of variation may be disregarded.

Capillary or Venous Blood.-Because of the discomfort of the procedures only two subjects provided simultaneous capillary and venous blood samples. The pairs of $H$ values were $(3 \cdot 8$, $3.4)$ and $(4 \cdot 7,4 \cdot 2)$ for capillary and venous samples respectively, which suggests that the difference, if any, is small.

Dose Level of Glucose.-By using data for 12 subjects reported by Castro et al. (1970) we found that $\mathrm{H}$ values are not systematically affected by changes in amounts of glucose ingested. The mean values of $H$ for the 12 subjects were $2 \cdot 6,2 \cdot 5,2 \cdot 6$, and $2 \cdot 6$ for glucose doses of $50,75,100 \mathrm{~g}$, and $1.75 \mathrm{~g} / \mathrm{kg}$ body weight respectively.

The Timing of Blood Samples.-We compared the sampling sequence of $0,15,30,45,60,90$, and 120 minutes with 0 , $30,60,90$, and 120 minutes obtained by disregarding observations at 15 and 45 minutes in the same group. The ratio of areas was in general hardly affected, the correlation over 100 tests being 0.92 , but there were seven occasions when the ratio differed greatly owing to the omission of the 15-minute sample. The location of the calculated time of peak glucose was somewhat more affected; 80 curves gave estimates within five minutes of each other, but in 15 curves the estimates differed by more than 15 minutes. As would be expected, the time to peak and the area to one hour are more accurately estimated when 15 and 45-minute samples are included. However, for routine clinical purposes or the retrospective analysis of clinical data only a small loss of accuracy will be incurred by using 30minute intervals throughout.

Length of Earlier Fasting.-By convention subjects generally fast for at least 12 hours before an O.G.T.T. In seven subjects the test was performed after that conventional fast and again, within a week, after a light breakfast of tea and toast two hours before the test. There was no difference between the $\mathrm{H}$ indices for the two tests.

Reproducibility.-Repeated estimates of $\mathrm{H}$ based on O.G.T.T.s several weeks apart show that the index may vary by 1 or occasionally 2 points when conditions are apparently the same. By comparison with the overall range of values, the reproducibility of the indices in practice appears to be satisfactory. Since the $\mathrm{H}$ index is a ratio it is independent of the absolute concentrations of glucose, which is a great advantage when comparing results from different laboratories.

\section{Discussion}

We must emphasize that the power of the $\mathrm{H}$ index to discriminate between cases classified on conventional biochemical criteria is not a valid test of its clinical usefulness, because the conventional criteria themselves are arbitrary. Complete agreement cannot be expected since $\mathrm{H}$ reflects the shape of the homeostatic response curve, whereas conventional criteria are based on selected glucose concentrations and are hardly influenced by shape. The fact that $\mathrm{H}$ does indeed discriminate quite well between cases classified by conventional criteria proves only that the index is clinically "realistic." Since it expresses the pattern of homeostatic response, on theoretical grounds it is likely to be a more valid means of evaluating O.G.T.T. data than arbitrarily-chosen levels of glucose concentration.

The $\mathrm{H}$ index provides a method for the analysis of the blood glucose response to an oral glucose load which has clinical meaning, is easy to calculate, is reproducible, and has the additional advantages of giving a simple numerical description of glucose homeostatic control which can be used to evaluate changes with time and which lends itself to easy statistical analysis; moreover, it is not influenced by such factors as the site of blood sampling, the laboratory method used, the size of the glucose load, and even whether or not the patient has fasted overnight. We have found it to be useful in assessing the relatively small changes in glucose tolerance that occur during pregnancy and after taking hormonal contraceptives, details of which will be published elsewhere.

\section{Appendix: The Calculation of $\mathbf{H}$}

$$
\mathrm{H}=\text { Time to peak } \times \frac{\text { area above fasting level }(0-2 \mathrm{hr})}{\text { area above fasting level }(0-1 \mathrm{hr})}
$$

The calculations are illustrated by references to four examples of O.G.T.T. data (see table), the second of which is also shown

Four Examples of Oral Glucose Tolerance Test Results on which Methods of Calculating $H$ are Based (see Appendix)

\begin{tabular}{|c|c|c|c|c|c|c|c|c|}
\hline \multirow[b]{2}{*}{ Example: } & \multicolumn{4}{|c|}{$\begin{array}{l}\text { Glucose Concentrations } \\
(\mathrm{mg} / 100 \mathrm{ml})\end{array}$} & \multicolumn{4}{|c|}{$\begin{array}{l}\text { Increments above Fasting } \\
(\mathrm{mg} / 100 \mathrm{ml})\end{array}$} \\
\hline & 1 & 2 & 3 & 4 & 1 & 2 & 3 & 4 \\
\hline $\begin{array}{l}\text { Times: } \\
\text { Minutes Units } \\
\mathbf{0} \\
\begin{array}{c}\mathbf{0} \\
\text { (Fasting) }\end{array}\end{array}$ & 80 & 80 & 100 & 100 & 0 & 0 & 0 & 0 \\
\hline $\begin{array}{rr}15 & 1 \\
30 & 2 \\
45 & 3 \\
60 & 4 \\
90 & 6 \\
120 & 8\end{array}$ & $\begin{array}{r}95 \\
125 \\
115 \\
105 \\
95 \\
90\end{array}$ & $\begin{array}{r}95 \\
120 \\
90 \\
75 \\
75 \\
90\end{array}$ & $\begin{array}{l}130 \\
150 \\
170 \\
190 \\
180 \\
150\end{array}$ & $\begin{array}{l}130 \\
150 \\
170 \\
190 \\
200 \\
180\end{array}$ & $\begin{array}{l}15^{*} \\
45^{*} \\
35^{*} \\
25 \\
15 \\
10\end{array}$ & $\begin{array}{l}15^{*} \\
40^{*} \\
10^{*} \\
-5 \\
-5 \\
10\end{array}$ & $\begin{array}{l}30 \\
50 \\
70^{*} \\
90^{*} \\
80^{*} \\
50\end{array}$ & $\begin{array}{r}30 \\
50 \\
70 \\
90^{*} \\
100^{*} \\
80^{*}\end{array}$ \\
\hline
\end{tabular}

* Values used in calculating time to peak. 
graphically (see fig. 4). Since blood glucose concentrations during an O.G.T.T. are usually measured at 15- or 30-minute intervals it simplifies the arithmetic to work in time units of 15 minutes each. As shown in the table, glucose levels at $0,15,30,45,60$, 90, and 120 minutes correspond to $0,1,2,3,4,6$, and 8 time units.

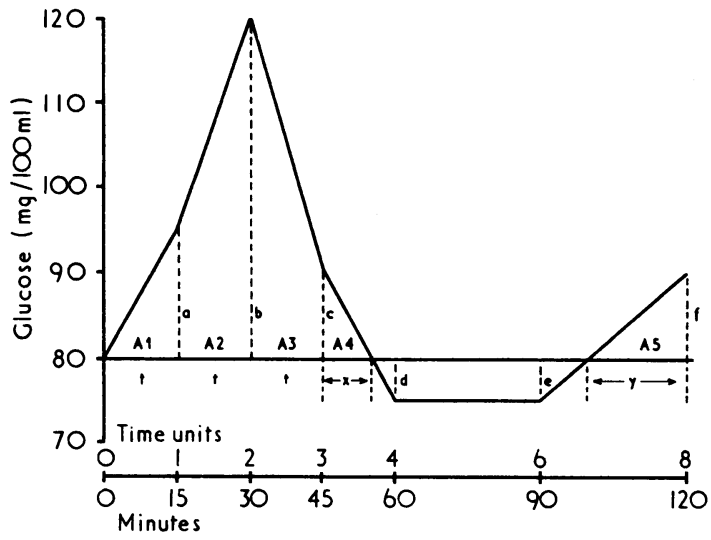

FIG. 4-Graphical representation of example 2.

\section{TIME TO PEAK}

This is calculated from three consecutive blood glucose concentrations, the middle value of which is the highest recorded value during a test; the time, in units, at which a parabola drawn through these points reaches a maximum is determined.

This calculation can be avoided by the use of a nomogram (fig. 5) as shown by the examples in the table.

Example 1.-The three values are 95,125 , and $115 \mathrm{mg} / 100 \mathrm{ml}$ and the two outside values differ from the highest by 30 and 10 respectively. The intervals between samples are one time unit, therefore the bold scale is used. Place a ruler at 30 on the left-

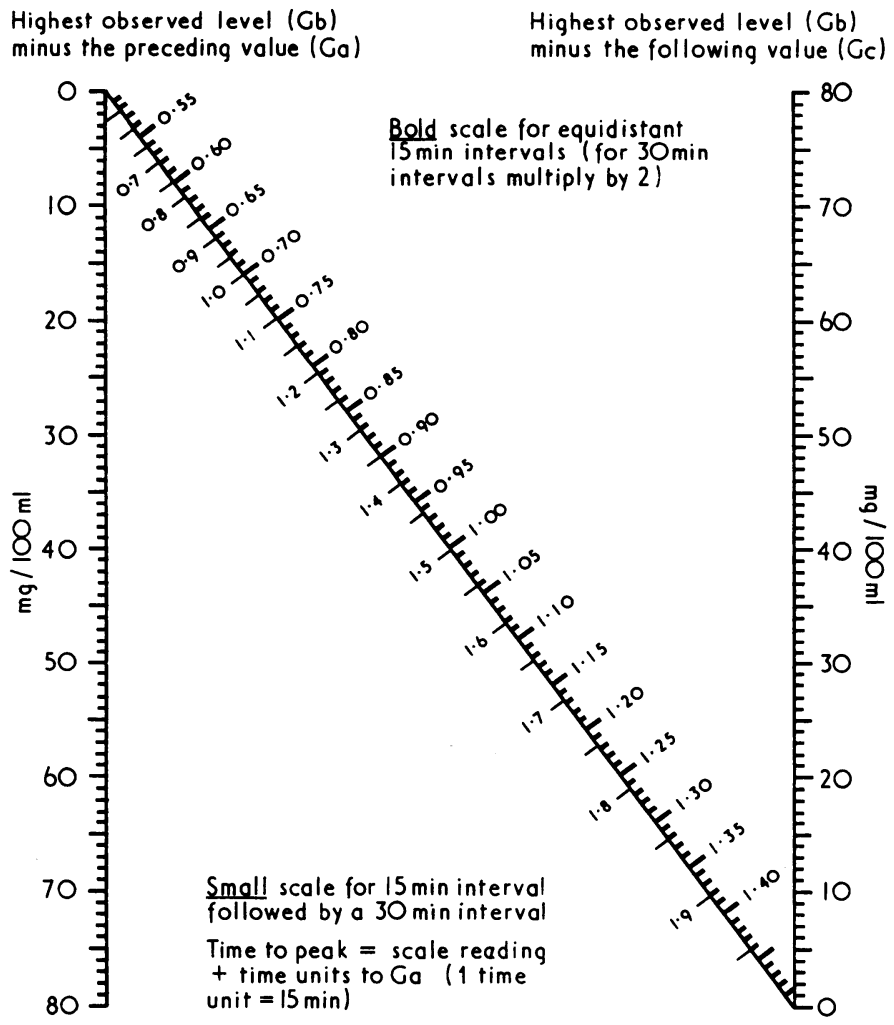

FIG. 5-A nomogram for the estimation of "time to peak." hand scale and at 10 on the right-hand scale; the ruler crosses the diagonal at 1.25 time units. To this must be added the number of time units at which the first of the three glucose values occurred; in this example therefore: time to peak = $1 \cdot 25+1=2 \cdot 25$. Similarly for example 2 we obtain: time to peak $=0.95+1=1.95$.

Example 3 illustrates a modification in procedure; the concentrations used are 170,190 , and $180 \mathrm{mg} / 100 \mathrm{ml}$, but since the first pair is separated by one time unit while the second pair is separated by two time units we use the small scale. The values required for reading the nomogram are 20 and 10 ; placing the ruler as described we read off 1.70 time units. Since the first of the three samples was at $45 \mathrm{~min}$ ( 3 time units), the time to peak $=1 \cdot 70+3=4 \cdot 70$.

In Example 4 the three glucose concentrations $(190,200$, and 180) are each separated by two time units, so we proceed as for example 1 but multiply the reading on the bold scale by 2 . The time to peak $=0.84 \times 2+4=5.68$.

Should the differences between the three concentrations used to estimate time to peak exceed the scale of the nomogram, divide both differences by the same number, say 2 , and then proceed as usual.

There are unusual situations where the nomogram cannot be used and the following conventions are adopted. (1) If the blood glucose concentration increases progressively to 120 minutes the time to peak is taken as 8 time units. (2) If there are three equal highest values time to peak is taken to be the mid-point of the two outer values.

There are occasional situations where the interpretation of the curve by use of the $\mathrm{H}$ index, as with more conventional criteria, is made difficult by delayed or uneven absorption of the glucose load from the gut-for instance, little or no increase in blood glucose concentration over the first 15 minutes with a rapid response thereafter, or when a response curve has a pronounced double peak. In such instances the O.G.T.T. should be repeated.

\section{AREAS ABOVE FASTING LEVELS}

The areas to be calculated comprise the sum of triangles and trapeziums above the fasting level; areas below the fasting level are ignored.

The concept may be understood more clearly by reference to fig. 4 where the incremental area during the first hour consists of $\mathrm{A} 1+\mathrm{A} 2+\mathrm{A} 3+\mathrm{A} 4$, to which $\mathrm{A} 5$ must be added to give the area for the first two hours. The bases of A1, A2 and $A 3$ are whole time units but the bases of A4, and A5 ( $x$ and $y$ ) are not and should be separately calculated. This presents no particular difficulty when the research worker wishes to calculate the total positive area with complete accuracy. Since glucose curves which fall below the fasting level in the course of a two-hour test signify good "glucose tolerance," for clinical purposes the calculation can be simplified by regarding all glucose concentrations less than the fasting level as zero increment. Adopting this principle, and referring to example 2 (fig. 4), the following general formulae can be applied.

(1) For sampling sequence $0,15,30,45,60,90$, and 120 minutes.

$\underline{\text { Area above fasting }(0-2 \mathrm{hr})}=2 \mathrm{a}+2 \mathrm{~b}+2 \mathrm{c}+3 \mathrm{~d}+4 \mathrm{e}+2 \mathrm{f}$ Area above fasting (0-1 hr) $=\frac{2 a+2 b+2 c+d}{2 a}$

(2) For a sampling sequence $0,30,60,90$, and 120 minutes. $\frac{\text { Area above fasting }(0-2 \mathrm{hr})}{\text { Area above fasting }(0-1 \mathrm{hr})}=\frac{2 \mathrm{~b}+2 \mathrm{~d}+2 \mathrm{e}+\mathrm{f}}{2 \mathrm{~b}+\mathrm{d}}$

Using formula (1) in conjunction with the increments shown in the table and remembering that any "negative increment" equals zero we obtain

Area above fasting $(0-2 \mathrm{hr})$

Area above fasting (0-1 hr) 


$$
\begin{aligned}
& \text { Example 1, } \frac{345}{215}=1.605 \\
& \text { Example 2, } \frac{150}{130}=1.154 \\
& \text { Example 3, } \frac{990}{390}=2.538 \\
& \text { Example 4, } \frac{1130}{390}=2.897
\end{aligned}
$$

\section{CALCULATION OF $\mathrm{H}$}

We have now all the necessary figures for the determination of $\mathrm{H}$.

Example $1, \mathrm{H}=2.25 \times 1.605=3.61$; example $2, \mathrm{H}=1.95$ $\times 1.154=2.25$; $\quad$ example $3, \quad \mathrm{H}=4.70 \times 2.538=11.93$; example $4, \mathrm{H}=5.68 \times 2.897=16.45$.

It may be pointed out that had we used the correct small triangles
A1 and A5 shown in fig. 5 instead of the simplified formula (1) the value of $\mathrm{H}$ for example 2 would have been $2 \cdot 16$.

We wish to express thanks to Professor A. M. Thomson and Professor F. E. Hytten for their help and advice during the preparation of this paper.

\section{References}

Ackerman, E., Rosevear, J. W., and McGuckin, W. F. (1964). Physics in Medicine and Biology, $9,202$.

Castro, A., Scott, J. P., Grettie, D. P., McFarlane, D., and Bailey, R. E. (1970). Diabetes, 19, 842

Ceresa, F., Serge, G., and Vitelli, A. (1962). Actualites Endocrinologiques, 3,

Fitzgeraid, M. G., and Keen, H. (1964). British Medical fournal, 1, 1568. Jarrett, R. J., and Graver, H. J. (1968). British Medical fournal, 2, 528. Lind, T., Van C. de Groot, H. A., Brown, G., and Cheyne, G. A., (1972). British Medical fournal, 3, 320

W.H.O. Expert Committee on Diabetes Mellitus (1965). World Health Organization. Technical Report Series, No. 310.

\title{
Effect of Carbohydrate Restriction in Obese Diabetics: Relationship of Control to Weight Loss
}

\author{
J. R. WALL， D. A. PYKE, W. G. OAKLEY
}

British Medical fournal, 1973, 1, 577-578

\section{Summary}

Two hundred newly diagnosed, fat diabetics were studied to determine whether control of the diabetes depended on loss of weight or on carbohydrate restriction alone. Satisfactory control was achieved by dietary treatment alone in $\mathbf{1 5 9}$ patients 30 required oral hypoglycaemic drugs and 11 were 18 of whom had actually gained weight. Of the remaining patients, 30 required oral hypoglycaemic drugs and 11 were still uncontrolled but not yet on other treatment.

The mean initial excess weight of all 200 patients was $28 \%$. Of those who remained uncontrolled on diet alone the mean initial excess weight $(31 \%)$ was slightly but not significantly greater than that of those who were controlled (27\%). Patients who were controlled lost slightly more weight than patients who were not, but their excess weight at the time of control was still $21 \%$.

We conclude that in $80 \%$ of obese diabetics control can be achieved by diet alone and that it is usually independent of weight loss.

\section{Introduction}

A large proportion of adult-onset diabetics are significantly overweight at the time of diagnosis (Pyke and Please, 1957). Although weight reduction is generally thought to be needed to achieve good diabetic control (Newburgh and Conn, 1939; Himsworth, 1949) we have noticed that many diabetics are controlled within days of beginning. carbohydrate restriction before significant weight reduction has occurred (Pyke, 1972).

We have therefore studied 200 obese adult-onset diabetics

Diabetic Department, King's College Hospital, London SE5 9RS

J. R. Wall, M.D., M.R.A.C.P., Clinical Assistant

D. A. Pyke, M.D., F.R.C.P., Physician

W. G. Oakley, M.D., F.R.C.P., Physician who were treated by diet alone to determine whether control of the diabetes depends on weight loss or on carbohydrate restriction alone.

\section{Patients and Methods}

The patients were newly diagnosed diabetics attending the outpatient clinic consecutively over the past three years, who were at least $10 \%$ above their expected weight* (mean excess $27.6 \%$ ). They had had no previous treatment. None of the patients was clinically dehydrated and there were no episodes of coma. There were 108 women and 92 men and their ages ranged from 23 to 90 , with over half in the 50-70 age group.

After the initial assessment and confirmation of the diagnosis the patients were started on a diet consisting of either carbohydrate restriction (usually to $100-150 \mathrm{~g}$ daily) or total restriction (usually to 1,000 calories). The diet was supervised at the first visit by the clinic dietician.

At each subsequent visit diabetic control was assessed from the presence and severity of symptoms, the degree of glycosuria, and the blood sugar levels. The patients were weighed under constant conditions and correlation was made between the control of the diabetes and the weight excess.

Final assessment was made either at the time of control or at the time that dietary management was thought to have failed and the patient was started on oral hypoglycaemic drugs. For this study we have not followed the patients after the time of first achieving control.

Diabetic control was defined as loss of symptoms, a blood sugar level under $160 \mathrm{mg} / 100 \mathrm{ml}$ about two hours after breakfast, and no more than a trace of glycosuria in one of two specimens (one from the previous night and one on the day of examination).

Blood glucose was estimated in whole blood by the ferricyanide method. Urine glucose was estimated with Clinitest.

- Taken from tables given in the New York Metropolitan Life Insurance Co. statistical bulletin November-December 1959. 